\title{
A phylum level analysis reveals lipoprotein biosynthesis to be a fundamental property of bacteria
}

\author{
lain C. Sutcliffe ${ }^{1 凶}$, Dean J. Harrington ${ }^{2}$, Matthew I. Hutchings ${ }^{3}$ \\ ${ }^{1}$ School of Life Sciences, University of Northumbria at Newcastle, Newcastle upon Tyne, NE1 8ST, UK \\ ${ }^{2}$ Division of Biomedical Science, School of Life Sciences, University of Bradford, West Yorkshire, BD7 1DP, UK \\ ${ }^{3}$ School of Biological Sciences, University of East Anglia, Norwich Research Park, Norwich, NR4 7TJ, UK \\ $\bowtie$ Correspondence: iain.sutcliffe@northumbria.ac.uk
}

Bacterial lipoproteins are proteins that are post-translationally modified with a diacylglyceride at an $\mathrm{N}$ terminal cysteine, which serves to tether these proteins to the outer face of the plasma membrane or to the outer membrane. This paper reviews recent insights into the enzymology of bacterial lipoprotein biosynthesis and localization. Moreover, we use bioinformatic analyses of bacterial lipoprotein signal peptide features and of the key biosynthetic enzymes to consider the distribution of lipoprotein biosynthesis at the phylum level. These analyses support the important conclusion that lipoprotein biosynthesis is a fundamental pathway utilized across the domain bacteria. Moreover, with the exception of a small number of sequences likely to derive from endosymbiont genomes, the enzymes of bacterial lipoprotein biosynthesis appear unique to bacteria, making this pathway an attractive target for the development of novel antimicrobials. Whilst lipoproteins with comparable signal peptide features are encoded in the genomes of Archaea, it is clear that these lipoproteins have a distinctive biosynthetic pathway that has yet to be characterized.

\section{INTRODUCTION}

The correct functioning of all biological membranes depends on the activities of both integral and peripheral membrane proteins. Of the latter, many proteins of bacterial, archaeal and eukaryotic cells can be localized to the surfaces of membranes by lipid modification. Bacterial lipoproteins (BaLpp) are a structurally unique class of proteins wherein membrane association is mediated by a diacylglycerol unit thioether linked to an $\mathrm{N}$-terminal cysteine (Braun and $\mathrm{Wu}$,
1994; Hutchings et al., 2009). In those bacteria with a single membrane (notably Actinobacteria, Firmicutes and Tenericutes), BaLpp are inevitably associated with the outer face of the plasma membrane. However, the majority of bacterial phyla are comprised of diderm bacteria (i.e. those that contain an outer membrane, typically based on lipopolysaccharide; Sutcliffe, 2010) and it is well established that BaLpp are translocated to the outer membrane as well. In this perspective we will consider new insights into the BaLpp biosynthetic pathway and how its distribution emphasizes the significance of BaLpp as fundamental components of bacteria.

\section{BIOSYNTHESIS OF BACTERIAL BALPP}

Protein lipid modification in bacteria occurs post-translationally, following translocation of the BaLpp precursor (pre-BaLpp) by either the Sec or the Tat translocation pathway (Hutchings et al., 2009; Shruthi et al., 2010; Thompson et al., 2010; Widdick et al., 2011). Pre-BaLpp are directed to either translocation pathway by the presence of specialized 'Type II' BaLpp signal peptides. As the TAT translocase transports fully folded proteins, the BaLpp biosynthetic machinery must be able to engage with folded proteins, most likely transiently membrane-anchored by their signal peptide. In the case of Sec-translocated BaLpp, it can be hypothesized that the BaLpp folds on emergence from the Sec translocon but it is not yet known whether lipidation precedes or follows protein folding. The defining feature of bacterial BaLpp signal peptides is the presence of a 'lipobox' sequence motif containing the invariant cysteine residue that is lipid modified (Rahman et al., 2008). Recognition of this motif, located in the context of other typical signal peptide features (i.e. a positively charged n-region and a hydrophobic h-regi- 
on), has allowed accurate bioinformatic methods for the prediction of proteins as BaLpp to be developed (Sutcliffe and Harrington, 2002; Juncker et al., 2003; Babu et al., 2006; Bagos et al., 2008; Rahman et al., 2008). These methods can be usefully applied to the prediction of putative BaLpp in whole genomes (for recent examples, see Remans et al., 2010; Thompson et al., 2010; Reffuveille et al., 2011). Notably, bioinformatic studies indicate that BaLpp are a numerically significant proportion (typically $1 \%-3 \%$ ) of virtually all bacterial proteomes that have been analyzed (Table 1) although they are more prevalent $(>10 \%)$ in some Bacteroidetes proteomes (Bendtsen et al., 2005; Babu et al., 2006).

The signal peptide 'lipobox' is the target sequence for pre-BaLpp lipid modification by prolipoprotein diacylglyceryol transferase (Lgt), the first enzyme in the BaLpp biosynthetic path. Lgt transfers a diacylglycerol moiety derived from membrane phosphatidylglycerol to BaLpp (Braun and Wu, 1994; Sankaran et al., 1995). Lgt is an integral membrane protein; bioinformatic analyses (unpublished observations) predict seven membrane spanning domains, with a significant periplasmic loop between TM4 and TM5 and an $\mathrm{N}$-out/C-in topology. These predictions are consistent with the topology mapping of Escherichia coli Lgt (Daley et al., 2005; Pailler et al., 2012), contradicting data suggesting that Lgt is a peripheral membrane protein that is readily extractable from E. coli inverted membrane vesicles (Selvan and Sankaran, 2008). The positioning of conserved residues in Lgt (unpublished observations; Pailler et al., 2012) is consistent with the enzyme utilizing membrane lipid substrates and interacting with the signal peptide lipobox at the interface at the outer surface of the plasma membrane. Information on the catalytic mechanism of Lgt action is limited but a number of key residues important to the activity of the $E$. coli enzyme have been identified, mainly located in two conserved motifs (R143G154 and Y235-E243, E. coli numbering [Qi et al., 1995; Sankaran et al., 1997; Pailler et al., 2012]). In addition to engaging with protein substrates from both the Sec and TAT translocases (see above), Lgt also appears to be able to process at least one lipoprotein that is inserted into the plasma membrane, in part, via the Sec-independent YidC pathway (van Bloois et al., 2006; Celebi et al., 2008), which likely also involves YidD (Yu et al., 2011).

Following pre-BaLpp lipid modification by Lgt, the signal peptide is removed by the dedicated lipoprotein signal peptidase, Lsp (Sankaran and Wu, 1995; Tjalsma et al., 1999). Lsp cleaves $\mathrm{N}$-terminal to the lipobox cysteine and the resulting diacylglceryl-modified $\mathrm{N}$-terminal cysteine is sufficient to anchor BaLpp to the membrane. Lsp is an integral membrane protein with four membrane spanning domains and an $\mathrm{N}$-in/C-in topology (Daley et al., 2005). Site directed mutagenesis of the Bacillus subtilis enzyme has implicated two conserved aspartic acid residues as being critical to the mechanism of Lsp (Tjalsma et al., 1999) and this has been confirmed for Streptomyces coelicolor Lsp (Thompson et al.,
2010). Lsp is the target of the cyclic depsipeptide antibiotic globomycin (Kiho et al., 2004), which has been a useful research tool for experimentally verifying BaLpp (e.g. Hutchings et al., 2006).

As noted above, the action of Lsp is sufficient to leave a diacyl-anchored BaLpp localized to the outer face of the plasma membrane. However, further BaLpp processing steps can also occur: a lipoprotein $\mathrm{N}$-acyltransferase, Lnt, can transfer a third amide linked fatty acid onto the $\mathrm{N}$-terminus of the BaLpp (Gupta and Wu, 1991; Sankaran et al., 1995). Topology modelling of Lnt has proven difficult and different models suggest either six or eight membrane spanning domains, although topology mapping of the $E$. coli enzyme suggested six (Robichon et al., 2005). In either model, a large periplasmic domain is predicted between the penultimate and the $\mathrm{C}$-terminal transmembrane helices. Elegant studies by the Buddelmeijer group have demonstrated that this catalytic domain belongs to the $\mathrm{CN}$ hydrolase superfamily and identified a two-step "ping-pong" reaction mechanism via a thio-ester acyl enzyme intermediate (Vidal-Ingigliardi et al., 2007; Buddelmeijer and Young, 2010; Hillmann et al., 2011). E. coli Lnt also exhibits a preference for phosphatidylethanolamine as substrate and its activity is also dependent on the acyl chain length of the phospholipid substrate (Hillmann et al., 2011). Conceivably the different predicted topologies of Lnt could reflect structural rearrangements associated with substrate interactions during the ping-pong reaction mechanism.

$\mathrm{N}$-acylation by Lnt is important in Proteobacteria as this step is a prerequisite for BaLpp translocation to the outer membrane via the Lol machinery (Fukuda et al., 2002; Robichon et al., 2005; see below). Recently, it has also been shown that Lnt homologues of Mycobacterium smegmatis, Mycobacterium tuberculosis and Streptomyces scabies are also functional $\mathrm{N}$-acyltransferases (Tschumi et al., 2009; Widdick et al., 2011) indicating that tri-acylation of BaLpp occurs in at least some Actinobacteria. Intriguingly, recent structural evidence has confirmed the presence of tri-acylated BaLpp in representative Firmicutes and Tenericutes despite the absence of obvious Int orthologues in the sequenced genomes of members of these phyla (Le Hénaff et al., 2002; Asanuma et al., 2011; Serebryakova et al., 2011). Consequently, it is likely that Lnt-independent mechanisms for bacterial protein $\mathrm{N}$-acylation await discovery. Elucidation of $\mathrm{N}$-acylation pathways in pathogenic bacteria is of considerable importance as it is clear that di-acyl and tri-acyl BaLpp interact differently with the human innate immune system (for example, see Schenk et al., 2009).

Downstream of the biosynthesis of BaLpp, it is likely that intramembrane proteases are responsible for further processing of the released signal peptides, thereby preventing their accumulation in the membrane (Suzuki et al., 1987; Chandler and Dunny, 2004; Denham et al., 2008). In some 
Table 1 Distribution of the BaLpp biosynthesis and Lol translocation pathways in the major phyla of bacteria

\begin{tabular}{|c|c|c|c|c|c|}
\hline \multirow{2}{*}{ Phylum } & \multirow{2}{*}{ BaLpp $^{1}$} & \multicolumn{4}{|c|}{ Protein (PFAM code) } \\
\hline & & Lgt (PF01790) & Lsp (PF01252) & LolA (PF03548) & LolB (PF03550) \\
\hline Acidobacteria & NA & 6 & 6 & 6 & 0 \\
\hline Actinobacteria $^{2}$ & $1.4 \%-2.9 \%(n=20)$ & 269 & 271 & 1 & 0 \\
\hline Aquifex & $1.2 \%(n=1)$ & 8 & 6 & 11 & 0 \\
\hline Bacteroidetes & $3.4 \%-12.6 \%(n=4)$ & 128 & 129 & 98 & 0 \\
\hline Chlamydia & $1.1 \%-2.7 \%(n=9)$ & 25 & 25 & 0 & 0 \\
\hline Chlorobi & $1.5 \%(n=1)$ & 12 & 12 & 0 & 0 \\
\hline Chloroflexi ${ }^{2}$ & $1.4 \%-1.8 \%(n=2)$ & 16 & 16 & 0 & 0 \\
\hline $\begin{array}{l}\text { Candidatus Cloacamonas } \\
\text { acidaminovorans WWE1 }\end{array}$ & NA & 1 & 1 & 1 & 0 \\
\hline Chrysiogenetes & NA & 1 & 1 & 1 & 0 \\
\hline Cyanobacteria & $0.6 \%-1.6 \%(n=10)$ & 60 & 57 & 0 & 0 \\
\hline Deferribacteres & NA & 3 & 2 & 3 & 0 \\
\hline Deinococcus-Thermus & $1.2 \%-1.7 \%(n=3)$ & 13 & 13 & 2 & 0 \\
\hline Dictyoglomi & NA & 2 & 2 & 0 & 0 \\
\hline Elusimicrobia & NA & 2 & 2 & 1 & 0 \\
\hline Fibrobacteres & NA & 1 & 1 & 1 & 0 \\
\hline Firmicutes $^{2}$ & $1.0 \%-3.7 \%(n=50)$ & 614 & 607 & 12 & 0 \\
\hline Fusobacteria & $1.9 \%(n=1)$ & 22 & 22 & 0 & 0 \\
\hline Gemmatimonadetes & NA & 1 & 1 & 1 & 0 \\
\hline Lentisphaerae & NA & 2 & 2 & 1 & 0 \\
\hline Nitrospirae & NA & 5 & 5 & 5 & 0 \\
\hline Planctomycetes & $1.2 \%(n=1)$ & 8 & 8 & 2 & 0 \\
\hline Poribacteria & NA & $N_{Y D}^{3}$ & $1^{4}$ & $N Y D^{3}$ & $N Y D^{3}$ \\
\hline Proteobacteria & $0.4 \%-6.7 \%(n=110)$ & 927 & 930 & 1236 & $832^{5}$ \\
\hline Spirochaetes & $0.5 \%-4.5 \%(n=6)$ & 37 & 38 & 24 & 0 \\
\hline Synergistites & NA & 4 & 2 & 0 & 0 \\
\hline Tenericutes $^{2}$ & $0.3 \%-6.4 \%(n=14)$ & 26 & 27 & 0 & 0 \\
\hline Thermodesulfobacteria & NA & $2^{4}$ & $2^{4}$ & $2^{4}$ & 0 \\
\hline Thermotoga & $1.0 \%(n=1)$ & 13 & 11 & 0 & 0 \\
\hline Verrucomicrobiae & NA & 8 & 8 & 2 & 0 \\
\hline Candidate NC10 & NA & 1 & 1 & 1 & 0 \\
\hline Candidate TG1 & NA & 1 & 1 & 0 & 0 \\
\hline Candidate TM7 & NA & $1^{4}$ & $1^{4}$ & 0 & 0 \\
\hline
\end{tabular}

$1 \%$ of proteome predicted to be BaLpp using LipoP. Bioinformatic data taken from Babu et al. (2006). $n$, number of genomes analysed at that time; NA, genome data not available in 2006.

${ }^{2}$ Monoderm phyla (Sutcliffe, 2010) and as such expected to lack the Lol pathway.

${ }^{3}$ NYD, not yet determined. The whole genome shotgun sequence of Candidatus Poribacteria sp. WGA-A3 is only ca. two thirds complete; Lgt is expected to be present as Lsp can be identified.

${ }^{4}$ Identified by BLAST analysis with appropriate $E$. coli sequences as query.

${ }^{5}$ Notably absent from all available $\alpha$ - or $\varepsilon$-Proteobacteria genomes. 
bacteria (such as enterococci) the released peptide products may play important roles as peptide pheromones (Chandler and Dunny, 2004).

\section{LIPOPROTEIN LOCALIZATION TO OUTER MEMBRANES}

As indicated above, many BaLpp in diderm bacteria appear to be targeted to the outer membrane by the Lol pathway, which has been extensively characterized in Proteobacteria by the Tokuda laboratory (Narita and Tokuda, 2006; Tokuda, 2009; Narita, 2011). In the Lol pathway, BaLpp destined for the outer membrane are extracted from the plasma membrane by the LolCDE ABC transport system (Tokuda, 2009; Narita, 2011), passed to the periplasmic shuttle protein LolA and then transferred to the outer membrane component LolB (itself a BaLpp orientated at the periplasmic face of the outer membrane). Detailed studies of the mechanism of transfer suggest that LolA binds the BaLpp lipid anchor into a hydrophobic pocket and then passes the substrate to the structurally related hydrophobic pocket of LolB in a "mouth-to-mouth" manner (Okuda and Tokuda, 2009). Subsequently, BaLpp are released to the inner leaflet of the outer membrane by an as yet unknown mechanism (Tsukahara et al., 2009). In some cases, BaLpp may also be translocated to the external surface of the outer membrane (see below).

It appears that most of the ca. 90 BaLpp encoded in the $E$. coli genome are targeted to the periplasmic face of the outer membrane by the Lol pathway, whereas a small number are retained at the plasma membrane. Discrimination of these different destinations for BaLpp is based on the presence of a 'Lol avoidance' signal in plasma membrane BaLpp, localized to the $\mathrm{N}$-terminus. The canonical signal is an Asp at position +2 but other amino acid signals extending to positions +3 and +4 are now also known (Lewenza et al., 2006; Narita and Tokuda, 2007; Lewenza et al., 2008). As noted above, N-acylation by Lnt must also precede BaLpp translocation to the outer membrane (Fukuda et al., 2002; Robichon et al., 2005). However it has recently been shown that overexpression of the LoICDE ABC transporter can bypass the requirement for $\mathrm{N}$-acylation in specific genetic backgrounds suggesting that, in these circumstances, the Lol pathway can localize di-acylated BaLpp (including Braun's lipoprotein) to the outer membrane (Narita and Tokuda, 2011). Importantly, this study indicates that the $E$. coli Lol pathway has a preference, rather than an absolute requirement, for tri-acylated BaLpp.

\section{DISTRIBUTION OF THE BALPP BIOSYNTHETIC PATHWAY}

Bioinformatic pattern searches that identify the signal peptide features of BaLpp have indicated that BaLpp are abundant in the predicted proteomes of bacteria from at least 15 phyla (Bendtsen et al., 2005; Babu et al., 2006; Setubal et al., 2006)
(Table 1). These analyses are extended by consideration of the distribution of Lgt and Lsp, the defining enzymes in the BaLpp biosynthetic pathway. Data from the PFAM (Table 1) and Interpro databases (not shown) clearly indicate the Lgt and Lsp proteins are encoded in the genomes of representatives of all bacterial phyla for which sequence data are available, with the exception of the whole genome shotgun sequence for Candidatus Poribacteria sp. WGA-A3. This genome contains an Isp but currently lacks an lgt homologue (most likely as only approximately two thirds of this genome has been sequenced). Most genomes contain only a single copy of each of these genes although bacteria of some phyla typically contain more than one Lgt (e.g. Acidobacteria; Chloroflexi) or Lsp (e.g. Chrysiogenetes; Gemmatimonadetes). It may be that in some organisms, specific BaLpp (or subsets) are processed by dedicated biosynthetic enzymes but this does not appear to be the case in Streptomyces coelicolor, the only organism with two Lgt that has been investigated to date (Thompson et al., 2010). In contrast, a Listeria monocytogenes EGD-e IspA mutant failed to process several pre-BaLpp and was defective for phagosomal escape in vitro (Réglier-Poupet et al., 2003), despite the presence of a second putative Lsp (Lmo1101) suggesting distinct roles for these two enzymes.

Cumulatively, the above data lead us to the important conclusion that BaLpp biosynthesis is a fundamental property of all bacterial cells, with the abundance of BaLpp encoded in genomes reflecting their functional diversity and physiological significance. Moreover, this pathway is almost unique to bacteria as the BaLpp biosynthetic enzymes are not encoded in the genomes of either Archaea (see below) or most sequenced Eukarya. However, with respect to the latter, it should be noted that bona fide Lgt and Lsp sequences are encoded in the chromatophore genome of Paulinella chromatophora which is derived from a cyanobacterial endosymbiont (Nowack et al., 2008). More unusually, the genome of Perkinsus marinus, a pathogenic alveolate protozoan, contains two tandem lgt coding sequences and two adjacent near identical $/ s p$ sequences which are divergently arranged. This may reflect the presence of a putative relic plastid in this organism (Joseph et al., 2010; Fernández Robledo et al., 2011). Intriguingly, however, one Lgt and Lsp were detected in an expressed sequence tag library, suggesting functionality of the pathway (Joseph et al., 2010).

The distribution of Lnt orthologues in bacteria is more difficult to gauge accurately as these proteins belong to the $\mathrm{CN}$ hydrolase superfamily that is universally distributed. The widespread distribution of Int homologues (data not shown), and the demonstration that Lnt orthologues are functional in representative Actinobacteria (see above) suggest that this third step in BaLpp biosynthesis operates widely. Nevertheless, it is well accepted that obvious Lnt orthologues are not encoded in the genomes of some taxa, notably Firmicutes and Tenericutes. However, the strong evidence for 
Lnt-independent $\mathrm{N}$-acylation of BaLpp in these taxa (see above) indicates that alternative mechanisms must exist for the synthesis of tri-acylated BaLpp and these pathways require further investigation. Cumulatively, these observations suggest that tri-acylation of BaLpp is likely a very widespread phenomenon.

Cell envelope organization at the phylum level reveals that most phyla are comprised of diderm bacteria (Sutcliffe, 2010). Thus it is surprising that the well characterized Lol pathway for translocation of BaLpp to the outer membrane is not well conserved at the phylum level (Table 1). The distribution of LolB is notably restricted, even within the Proteobacteria (being absent from the genomes of $\alpha$ - and $\varepsilon$-Proteobacteria), as noted previously (Narita and Tokuda, 2006; Narita, 2011). LolA is more widely distributed but still apparently absent from many diderm phyla (Table 1). Moreover, clear orthologues of LolE cannot be identified in some divisions of the Proteobacteria (Narita and Tokuda, 2006; Narita, 2011). These data suggest that variant or alternative pathways exist for the translocation of the BaLpp across the periplasm and/or into the outer membranes of many bacteria. Variation on the terminal stage of the Lol pathway has been studied in the phylum Spirochaetes (particularly Borellia spp.) where $\mathrm{N}$-terminal 'tether' regions direct BaLpp to the surface of the outer membrane (Schulze and Zückert, 2006; Kumru et al., 2011). Although LolA homologues are encoded in spirochete genomes, LolB is absent. Thus the factors mediating surface localization of BaLpp are not yet understood, although the C-termini of the unfolded BaLpp may be important (Schulze et al., 2010). Mutation of LolA in Flavobacterium johnsoniae (phylum Bacteroidetes) abrogates gliding motility of this bacterium consistent with a role of LolA in the localization of several BaLpp known to contribute to the gliding mechanism (Rhodes et al., 2011).

\section{PROTEIN LIPIDATION IN THE DOMAIN ARCHAEA}

It is a significant conclusion of the present study that post-translational diacylglycerol modification of BaLpp by Lgt and $L s p$ represents a fundamental (and near unique) property of bacteria. Bioinformatic analyses using either PFAM (see above) or BLAST homology searches (against 106 archaeal genomes) has consistently failed to detect archaeal orthologues of Lgt and Lsp. Nevertheless, bioinformatic analyses of archaeal genomes have readily detected encoded proteins with 'Type II' (lipobox containing) signal peptides (Bardy et al., 2003; Saleh et al., 2010; Storf et al., 2010). These putative archaeal lipoproteins (ArLpp) are confidently predicted to be lipoproteins by reliable tools such as LipoP and PredLipo (Juncker et al., 2003; Bagos et al., 2008) and, as is the case for BaLpp, their normal localization can be disrupted by site directed mutagenesis of the lipobox cysteine suggesting this to be a critical determinant of signal peptide processing (Giménez et al., 2007; Storf et al., 2010). More- over, a mass spectrometry analysis of a halocyanin from Natronomonas (formerly Natronobacterium) pharaonis was consistent with the lipid modification of the canonical lipobox cysteine with a diphytanyl (glycerol)diether prior to signal peptide cleavage (Mattar et al., 1994). Cumulatively, these observations suggest that there is an Lgt/Lsp independent pathway for post-translational cysteine lipidation of ArLpp. The differing enzymology of this pathway presumably reflects the distinctive lipid composition of archaeal membranes and thus the nature of the lipid anchor added. It remains striking that this pathway still appears to depend on conserved signal peptide characteristics and this raises intriguing questions about the timing of the evolution of these signal peptides. As in bacteria, it appears that archaeal genomes encode ArLpp with signal peptides that direct translocation by either the Sec or Tat pathways (Giménez et al., 2007; Storf et al., 2010) and in some haloarchaea it appears that the majority of ArLpp are putative Tat substrates (Storf et al., 2010). Recent analyses of a range of archaeal genomes suggest that the proportion of ArLpp they encode varies considerably between species, from few, if any, per genome (e.g. Pyrobaculum spp.) to many (e.g. $2.7 \%$ of the predicted proteome of $N$. pharaonis) (Saleh et al., 2010; Storf et al., 2010). As in monoderm bacteria, it is noted that solute-binding proteins of $A B C$ transport systems may be a functionally important category of ArLpp. Clearly identification of the biosynthetic pathway and chemical characterization of ArLpp remain an important area for future work.

\section{BaLpp biosynthesis as a novel drug target}

The apparent near uniqueness of the BaLpp biosynthetic pathway suggests that this pathway could present an attractive target for novel antibiotics. This view is also supported by the apparent essentiality of Lgt, Lsp and Lnt in those Proteobacteria studied to date, which most likely reflects the roles of outer membrane BaLpp in several key steps in outer membrane biosynthesis (Tokuda, 2009). There may however be limitations to the extent to which the different steps of the BaLpp biosynthetic pathway may provide realistic drug targets. The antibiotic globomycin inhibits Lsp but remains too toxic for clinical use and has yet to be derivatized into second generation antibiotics (Kiho et al., 2004). Significantly, a novel antimicrobial MAC13243 has recently been discovered that is able to inhibit the Lol pathway by binding to LolA (Pathania et al., 2009), again consistent with the importance of outer membrane BaLpp to diderm bacteria. Other Lol pathway inhibitors have also been reported (Ito et al., 2007). Targeting the activity of Lgt may prove less attractive since although inhibiting lipidation is likely to result in aberrant localization and functioning of BaLpp, it is also known that BaLpp are important in pathogen recognition by the host (Schenk et al., 2009). Indeed, in some animal models of disease lgt mutants have been suggested to have hypervirulent pheno- 
types (Bubeck Wardenburg et al., 2006; Henneke et al., 2008) but the significance of these findings remains to be determined. Thus, it may be that the steps downstream of Lgt are the most promising targets for novel antibiotics, particularly against pathogenic Proteobacteria. Moreover, inhibition of BaLpp biosynthesis in the endosymbionts of filarial nematodes may be a target for prevention of diseases caused by these globally significant parasites (Johnston et al., 2010).

\section{CONCLUDING COMMENTS}

Excellent progress has been made in defining the genetic and biochemical basis of BaLpp biosynthesis and, in Proteobacteria, translocation of BaLpp to the outer membrane. Comparative genomic analyses of the distribution of both the putative BaLpp and of the defining biosynthetic enzymes, Lgt and Lsp, reveal that BaLpp represent fundamental entities in the biology of bacteria. Whilst the Lgt and Lsp steps in the biosynthetic pathway appear universally conserved across bacterial phyla, further studies are needed to explore the extent to which different or variant mechanisms of $\mathrm{N}$-terminal acylation and outer membrane translocation operate. Structural studies of the various enzymes in the BaLpp biosynthetic pathway are needed but this is complicated by the membrane localization of these proteins. Such studies may be important in defining BaLpp biosynthesis as a novel antibiotic target. Moreover, further studies are clearly needed to investigate putative ArLpp biogenesis in Archaea.

\section{REFERENCES}

Asanuma, M., Kurokawa, K., Ichikawa, R., Ryu, K.-H., Chae, J.-H., Dohmae, N., Lee, B.L., and Nakayama, H. (2011). Structural evidence of $\alpha$-aminoacylated lipoproteins of Staphylococcus aureus. FEBS J 278, 716-728.

Babu, M.M., Priya, M.L., Selvan, A.T., Madera, M., Gough, J., Aravind, L., and Sankaran, K. (2006). A database of bacterial lipoproteins (DOLOP) with functional assignments to predicted lipoproteins. J Bacteriol 188, 2761-2773.

Bagos, P.G., Tsirigos, K.D., Liakopoulos, T.D., and Hamodrakas, S.J. (2008). Prediction of lipoprotein signal peptides in Gram-positive bacteria with a Hidden Markov Model. J Proteome Res 7, 5082-5093.

Bardy, S.L., Eichler, J., and Jarrell, K.F. (2003). Archaeal signal peptides-a comparative survey at the genome level. Protein Sci 12, 1833-1843.

Bendtsen, J.D., Binnewies, T.T., Hallin, P.F., Sicheritz-Pontén, T., and Ussery, D.W. (2005). Genome update: prediction of secreted proteins in 225 bacterial proteomes. Microbiology 151, 1725-1727.

Braun, V., and Wu, H.C. (1994). Lipoproteins: structure function, biosynthesis and model for protein export. New Comp. Biochem. 27, 319-341.

Bubeck Wardenburg, J., Williams, W.A., and Missiakas, D. (2006). Host defenses against Staphylococcus aureus infection require recognition of bacterial lipoproteins. Proc Natl Acad Sci U S A 103 13831-13836.

Buddelmeijer, N., and Young, R. (2010). The essential Escherichia coli apolipoprotein $\mathrm{N}$-acyltransferase (Lnt) exists as an extracytoplasmic thioester acyl-enzyme intermediate. Biochemistry 49, 341-346.

Celebi, N., Dalbey, R.E., and Yuan, J. (2008). Mechanism and hydrophobic forces driving membrane protein insertion of subunit II of cytochrome $\mathrm{bo}_{3}$ oxidase. J Mol Biol 375, 1282-1292.

Chandler, J.R., and Dunny, G.M. (2004). Enterococcal peptide sex pheromones: synthesis and control of biological activity. Peptides 25, 1377-1388.

Daley, D.O., Rapp, M., Granseth, E., Melén, K., Drew, D., and von Heijne, G. (2005). Global topology analysis of the Escherichia coli inner membrane proteome. Science 308, 1321-1323.

Denham, E.L., Ward, P.N., and Leigh, J.A. (2008). Lipoprotein signal peptides are processed by Lsp and Eep of Streptococcus uberis. J Bacteriol 190, 4641-4647.

Fernández Robledo, J.A., Caler, E., Matsuzaki, M., Keeling, P.J., Shanmugam, D., Roos, D.S., and Vasta, G.R. (2011). The search for the missing link: a relic plastid in Perkinsus? Int J Parasitol 41, 1217-1229.

Fukuda, A., Matsuyama, S., Hara, T., Nakayama, J., Nagasawa, H., and Tokuda, H. (2002). Aminoacylation of the $\mathrm{N}$-terminal cysteine is essential for Lol-dependent release of lipoproteins from membranes but does not depend on lipoprotein sorting signals. J Biol Chem 277, 43512-43518.

Giménez, M.I., Dilks, K., and Pohlschröder, M. (2007). Haloferax volcanii twin-arginine translocation substates include secreted soluble, C-terminally anchored and lipoproteins. Mol Microbiol 66, 1597-1606.

Gupta, S.D., and Wu, H.C. (1991). Identification and subcellular localization of apolipoprotein $\mathrm{N}$-acyltransferase in Escherichia coli. FEMS Microbiol Lett 62, 37-41.

Henneke, P., Dramsi, S., Mancuso, G., Chraibi, K., Pellegrini, E., Theilacker, C., Hübner, J., Santos-Sierra, S., Teti, G., Golenbock, D.T., et al. (2008). Lipoproteins are critical TLR2 activating toxins in group B streptococcal sepsis. J Immunol 180, 6149-6158.

Hillmann, F., Argentini, M., and Buddelmeijer, N. (2011). Kinetics and phospholipid specificity of apolipoprotein $N$-acyltransferase. J Biol Chem 286, 27936-27946.

Hutchings, M.I., Hong, H.-J., Leibovitz, E., Sutcliffe, I.C., and Buttner, M.J. (2006). The CseBC- $\sigma^{E}$ cell envelope stress signal transduction system of Streptomyces coelicolor is modulated by a novel lipoprotein. CseA. J. Bacteriol. 188, 7222-7229.

Hutchings, M.I., Palmer, T., Harrington, D.J., and Sutcliffe, I.C. (2009). Lipoprotein biogenesis in Gram-positive bacteria: knowing when to hold 'em, knowing when to fold 'em. Trends Microbiol 17, 13-21.

Ito, H., Ura, A., Oyamada, Y., Yoshida, H., Yamagishi, J., Narita, S., Matsuyama, S., and Tokuda, H. (2007). A new screening method to identify inhibitors of the Lol (localization of lipoproteins) system, a novel antibacterial target. Microbiol Immunol 51, 263-270.

Johnston, K.L., Wu, B., Guimarães, A., Ford, L., Slatko, B.E., and Taylor, M.J. (2010). Lipoprotein biosynthesis as a target for anti-Wolbachia treatment of filarial nematodes. Parasit Vectors 3 , 99.

Joseph, S.J., Fernández-Robledo, J.A., Gardner, M.J., El-Sayed, 
N.M., Kuo, C.-H., Schott, E.J., Wang, H., Kissinger, J.C., and Vasta, G.R. (2010). The Alveolate Perkinsus marinus: biological insights from EST gene discovery. BMC Genomics 11, 228.

Juncker, A.S., Willenbrock, H., Von Heijne, G., Brunak, S., Nielsen, H., and Krogh, A. (2003). Prediction of lipoprotein signal peptides in Gram-negative bacteria. Protein Sci 12, 1652-1662.

Kiho, T., Nakayama, M., Yasuda, K., Miyakoshi, S., Inukai, M., and Kogen, H. (2004). Structure-activity relationships of globomycin analogues as antibiotics. Bioorg Med Chem 12, 337-361.

Kumru, O.S., Schulze, R.J., Rodnin, M.V., Ladokhin, A.S., and Zückert, W.R. (2011). Surface localization determinants of Borrelia OspC/Vsp family lipoproteins. J Bacteriol 193, 2814-2825.

Le Hénaff, M., Crémet, J.Y., and Fontenelle, C. (2002). Purification and characterization of the major lipoprotein (P28) of Spiroplasma apis. Protein Expr Purif 24, 489-496.

Lewenza, S., Mhlanga, M.M., and Pugsley, A.P. (2008). Novel inner membrane retention signals in Pseudomonas aeruginosa lipoproteins. J Bacteriol 190, 6119-6125.

Lewenza, S., Vidal-Ingigliardi, D., and Pugsley, A.P. (2006). Direct visualization of red fluorescent lipoproteins indicates conservation of the membrane sorting rules in the family Enterobacteriaceae. J Bacteriol 188, 3516-3524.

Mattar, S., Scharf, B., Kent, S.B.H., Rodewald, K., Oesterhelt, D., and Engelhard, M. (1994). The primary structure of halocyanin, an archaeal blue copper protein, predicts a lipid anchor for membrane fixation. J Biol Chem 269, 14939-14945.

Narita, S.-I. (2011). ABC transporters involved in the biogenesis of the outer membrane in gram-negative bacteria. Biosci Biotechnol Biochem 75, 1044-1054.

Narita, S.-I., and Tokuda, H. (2006). An ABC transporter mediating the membrane detachment of bacterial lipoproteins depending on their sorting signals. FEBS Lett 580, 1164-1170.

Narita, S.-I., and Tokuda, H. (2007). Amino acids at positions 3 and 4 determine the membrane specificity of Pseudomonas aeruginosa lipoproteins. J Biol Chem 282, 13372-13378.

Narita, S.-I., and Tokuda, H. (2011). Overexpression of LolCDE allows deletion of the Escherichia coli gene encoding apolipoprotein N-acyltransferase. J Bacteriol 193, 4832-4840.

Nowack, E.C.M., Melkonian, M., and Glöckner, G. (2008). Chromatophore genome sequence of Paulinella sheds light on acquisition of photosynthesis by eukaryotes. Curr Biol 18, 410-418.

Okuda, S., and Tokuda, H. (2009). Model of mouth-to-mouth transfer of bacterial lipoproteins through inner membrane LolC, periplasmic LolA, and outer membrane LolB. Proc Natl Acad Sci U S A 106, 5877-5882.

Pailler, J., Aucher, W., Pires, M., and Buddelmeijer, N. (2012). Phosphatidylglycerol:prolipoprotein diacylglyceryl transferase (Lgt) of $\mathrm{E}$. coli has seven transmembrane segments and its essential residues are embedded in the membrane. $\mathrm{J}$ Bacteriol. doi:10.1128/JB.06641-11.

Pathania, R., Zlitni, S., Barker, C., Das, R., Gerritsma, D.A., Lebert, J., Awuah, E., Melacini, G., Capretta, F.A., and Brown, E.D. (2009). Chemical genomics in Escherichia coli identifies an inhibitor of bacterial lipoprotein targeting. Nat Chem Biol 5, 849-856.

Qi, H.-Y., Sankaran, K., Gan, K., and Wu, H.C. (1995). Structure-function relationship of bacterial prolipoprotein diacylglyceryl transferase: functionally significant conserved regions. J Bacteriol $177,6820-6824$.
Rahman, O., Cummings, S.P., Harrington, D.J., and Sutcliffe, I.C. (2008). Methods for the bioinformatic identification of bacterial lipoproteins encoded in the genomes of Gram-positive bacteria. World J Microbiol Biotechnol 24, 2377-2382.

Reffuveille, F., Leneveu, C., Chevalier, S., Auffray, Y., and Rincé, A. (2011). Lipoproteins of Enterococcus faecalis: bioinformatic identification, expression analysis and relation to virulence. Microbiology 157, 3001-3013.

Réglier-Poupet, H., Frehel, C., Dubail, I., Beretti, J.-L., Berche, P., Charbit, A., and Raynaud, C. (2003). Maturation of lipoproteins by type II signal peptidase is required for phagosomal escape of Listeria monocytogenes. J Biol Chem 278, 49469-49477.

Remans, K., Vercammen, K., Bodilis, J., and Cornelis, P. (2010). Genome-wide analysis and literature-based survey of lipoproteins in Pseudomonas aeruginosa. Microbiology 156, 2597-2607.

Rhodes, R.G., Samarasam, M.N., Van Groll, E.J., and McBride, M.J. (2011). Mutations in Flavobacterium johnsoniae sprE result in defects in gliding motility and protein secretion. J Bacteriol 193, 5322-5327.

Robichon, C., Vidal-Ingigliardi, D., and Pugsley, A.P. (2005). Depletion of apolipoprotein $\mathrm{N}$-acyltransferase causes mislocalization of outer membrane lipoproteins in Escherichia coli. J Biol Chem 280, 974-983.

Saleh, M., Song, C., Nasserulla, S., and Leduc, L.G. (2010). Indicators from archaeal secretomes. Microbiol Res 165, 1-10.

Sankaran, K., Gan, K., Rash, B., Qi, H.-Y., Wu, H.C., and Rick, P.D. (1997). Roles of histidine-103 and tyrosine-235 in the function of the prolipoprotein diacylglyceryl transferase of Escherichia coli. J Bacteriol 179, 2944-2948.

Sankaran, K., Gupta, S.D., and Wu, H.C. (1995). Modification of bacterial lipoproteins. Methods Enzymol 250, 683-697.

Sankaran, K., and Wu, H.C. (1995). Bacterial prolipoprotein signal peptidase. Methods Enzymol 248, 169-180.

Schenk, M., Belisle, J.T., and Modlin, R.L. (2009). TLR2 looks at lipoproteins. Immunity 31, 847-849.

Schulze, R.J., Chen, S.Y., Kumru, O.S., and Zückert, W.R. (2010). Translocation of Borrelia burgdorferi surface lipoprotein OspA through the outer membrane requires an unfolded conformation and can initiate at the C-terminus. Mol Microbiol 76, 1266-1278.

Schulze, R.J., and Zückert, W.R. (2006). Borrelia burgdorferi lipoproteins are secreted to the outer surface by default. Mol Microbiol 59, 1473-1484.

Selvan, A.T., and Sankaran, K. (2008). Localization and characterization of prolipoprotein diacylglyceryl transferase (Lgt) critical in bacterial lipoprotein biosynthesis. Biochimie 90, 1647-1655.

Serebryakova, M.V., Demina, I.A., Galyamina, M.A., Kondratov, I.G., Ladygina, V.G., and Govorun, V.M. (2011). The acylation state of surface lipoproteins of mollicute Acholeplasma laidlawii. J Biol Chem 286, 22769-22776.

Setubal, J.C., Reis, M., Matsunaga, J., and Haake, D.A. (2006). Lipoprotein computational prediction in spirochaetal genomes. Microbiology 152, 113-121.

Shruthi, H., Babu, M.M., and Sankaran, K. (2010). TAT-pathway-dependent lipoproteins as a niche-based adaptation in prokaryotes. J Mol Evol 70, 359-370.

Storf, S., Pfeiffer, F., Dilks, K., Chen, Z.Q., Imam, S., and Pohlschröder, M. (2010). Mutational and bioinformatic analysis of haloarchaeal lipobox-containing proteins. Archaea 2010, 410975. 
Sutcliffe, I.C. (2010). A phylum level perspective on bacterial cell envelope architecture. Trends Microbiol 18, 464-470.

Sutcliffe, I.C., and Harrington, D.J. (2002). Pattern searches for the identification of putative lipoprotein genes in Gram-positive bacterial genomes. Microbiology 148, 2065-2077.

Suzuki, T., Itoh, A., Ichihara, S., and Mizushima, S. (1987). Characterization of the sppA gene coding for protease IV, a signal peptide peptidase of Escherichia coli. J Bacteriol 169, 2523-2528.

Thompson, B.J., Widdick, D.A., Hicks, M.G., Chandra, G., Sutcliffe, I.C., Palmer, T., and Hutchings, M.I. (2010). Investigating lipoprotein biogenesis and function in the model Gram-positive bacterium Streptomyces coelicolor. Mol Microbiol 77, 943-957.

Tjalsma, H., Zanen, G., Venema, G., Bron, S., and van Dijl, J.M. (1999). The potential active site of the lipoprotein-specific (type II) signal peptidase of Bacillus subtilis. J Biol Chem 274, 28191-28197.

Tokuda, H. (2009). Biogenesis of outer membranes in Gram-negative bacteria. Biosci Biotechnol Biochem 73, 465-473.

Tschumi, A., Nai, C., Auchli, Y., Hunziker, P., Gehrig, P., Keller, P., Grau, T., and Sander, P. (2009). Identification of apolipoprotein $\mathrm{N}$-acyltransferase (Lnt) in mycobacteria. J Biol Chem 284,
27146-27156.

Tsukahara, J., Mukaiyama, K., Okuda, S., Narita, S.-I., and Tokuda, H. (2009). Dissection of LolB function-lipoprotein binding, membrane targeting and incorporation of lipoproteins into lipid bilayers. FEBS J 276, 4496-4504.

van Bloois, E., Haan, G.-J., de Gier, J.-W., Oudega, B., and Luirink, J. (2006). Distinct requirements for translocation of the $\mathrm{N}$-tail and C-tail of the Escherichia coli inner membrane protein CyoA. J Biol Chem 281, 10002-10009.

Vidal-Ingigliardi, D., Lewenza, S., and Buddelmeijer, N. (2007). Identification of essential residues in apolipoprotein $\mathrm{N}$-acyl transferase, a member of the CN hydrolase family. J Bacteriol 189, 4456-4464.

Widdick, D.A., Hicks, M.G., Thompson, B.J., Tschumi, A., Chandra, G., Sutcliffe, I.C., Brülle, J.K., Sander, P., Palmer, T., and Hutchings, M.I. (2011). Dissecting the complete lipoprotein biogenesis pathway in Streptomyces scabies. Mol Microbiol 80, 1395-1412.

Yu, Z., Lavèn, M., Klepsch, M., de Gier, J.-W., Bitter, W., van Ulsen, P., and Luirink, J. (2011). Role for Escherichia coli YidD in membrane protein insertion. J Bacteriol 193, 5242-5251. 